\title{
0. Bezeichnungen und Symbole
}

\section{Allgemeine Festlegungen}

$\underline{X}(\omega)$

FOURIER-Transformierte von $X(t)$

KK konjugiert komplexer Anteil einer davor angegebenen Größe; z. B.

$$
\begin{aligned}
& X(\omega)=\int_{-\infty}^{\infty} \mathrm{d} t X(t) \mathrm{e}^{-\mathrm{i} \omega t} \\
& X(t)=\frac{1}{2 \pi} \int_{-\infty}^{\infty} \mathrm{d} \omega \underline{X}(\omega) \mathrm{e}^{\mathrm{i} \omega t}
\end{aligned}
$$

$\langle X\rangle \quad \bar{X}=\frac{1}{u} \int_{-u / 2}^{u / 2} X(t) \mathrm{d} t \quad$ zeitlicher Mittelwert einer Größe über das Zeitintervall $u$ gilt $X+\mathrm{KK}=X+X^{*}$

$\begin{array}{ll}\approx & \text { angenähert } \\ \simeq & \text { von gleicher Größenordnung } \\ \lesssim & \text { kleiner oder angenähert gleich } . \\ \gtrsim & \text { größer oder angenähert gleich } \\ \ll & \text { klein gegen } \\ & \text { groß gegen }\end{array}$

\section{Elektrische Feldstärke und Polarisation}

$$
\begin{array}{ll}
\vec{E}(\vec{r}, t) & \text { elektrische Feldstärke } \\
\vec{e} & \text { Einheitsvektor in Feldstärkerichtung } \\
\hat{E}(\vec{r}, t) & \text { Schwingungsamplitude der elektrischen Feldstärke } \\
& \vec{E}(\vec{r}, t)=\frac{1}{2} \hat{E}(\vec{r}, t) \vec{e} \mathrm{e}^{\mathrm{i} \omega t}+\mathrm{KK} \\
A(\vec{r}, t) & \text { Wellenamplitude der elektrischen Feldstärke } \\
& \vec{E}(\vec{r}, t)=\frac{1}{2} A(\vec{r}, t) \vec{e} \mathrm{e}^{\mathrm{i}(\omega t-\vec{k} \vec{r})}+\mathrm{KK}
\end{array}
$$




$\begin{array}{ll}\vec{P}(\vec{r}, t) & \begin{array}{l}\text { Polarisation } \\ \vec{e}^{\prime}\end{array} \\ \bar{P}(\vec{r}, \mathrm{t}) & \text { Winheitsvektor in Polarisationsrichtung } \\ & \text { Wellenamplitude der Polarisation } \\ & \vec{P}(\vec{r}, t)=\frac{1}{2} \bar{P}(\vec{r}, t) \vec{e}^{\prime} \mathrm{e}^{\mathrm{i}(\omega t-\vec{k} \vec{r})}+\mathrm{KK}\end{array}$

\section{Einzelne häufig auftretende Symbole}

$A_{i j}$
$B_{i j}$
$c$
$\mathscr{E}$
$\mathscr{E}_{\mathrm{S}}$
$e$
$g$
$G$
$g_{\mathrm{V}}$
$g=g_{\mathrm{V}} \cdot L=\ln G$
$h, \hbar$
$\mathrm{i}$
$I$

$I_{\mathrm{S}}$

$\vec{k}$

$k=2 \pi / \lambda$

$k$

$k_{\mathrm{A}}$

$k_{\mathrm{B}}$

N

$N_{i}$

$\mathscr{N}$

$n$

$\vec{r}$

$\vec{r}=(x, y, z)$

$R$

$\mathscr{T}$

$T_{i j}$

$t$

$U$
Enstein-Koeffizient für spontane Emission zum Ubergang $i \rightarrow j$ Einstern-Koeffizient für Absorption $(i<j)$ bzw. induzierte Emission $(i>j)$

Vakuumlichtgeschwindigkeit

Energie

Sättigungsphotonenzahl pro Fläche

(Sättigungsenergie pro Fläche/Photonenenergie $\hbar \omega$ )

elektrische Elementarladung $(e>0)$

Linienform-Funktion

Verstärkung $\left(I_{\text {Ausg }}=I_{\text {Eing }} G\right)$

Verstärkungskoeffizient

( $L-$ Verstärkungslänge)

Wirkungsquantum, $\hbar=h / 2 \pi$

imaginäre Einheit

Photonenzahlflußdichte

( $\triangle$ Energiestromdichte/Photonenenergie $\hbar \omega$ )

Sättigungsphotonenflußdichte

(Sättigungsintensität/Photonenenergie $\hbar \omega$ )

Wellenzahlvektor

Wellenzahl

Ratenkonstante

Absorptionskoeffizient

BolTzMANN-Konstante

Anzahldichte

Besetzungszahldichte des $i$-ten Niveaus

Anzahl

Brechzahl

Ortskoordinate

Reflexionsvermögen

Temperatur

Energierelaxationszeit (longitudinale Relaxationszeit) zum Ubergang $i \rightarrow j$

Zeit

Strahlungsenergie pro Volumeneinheit 


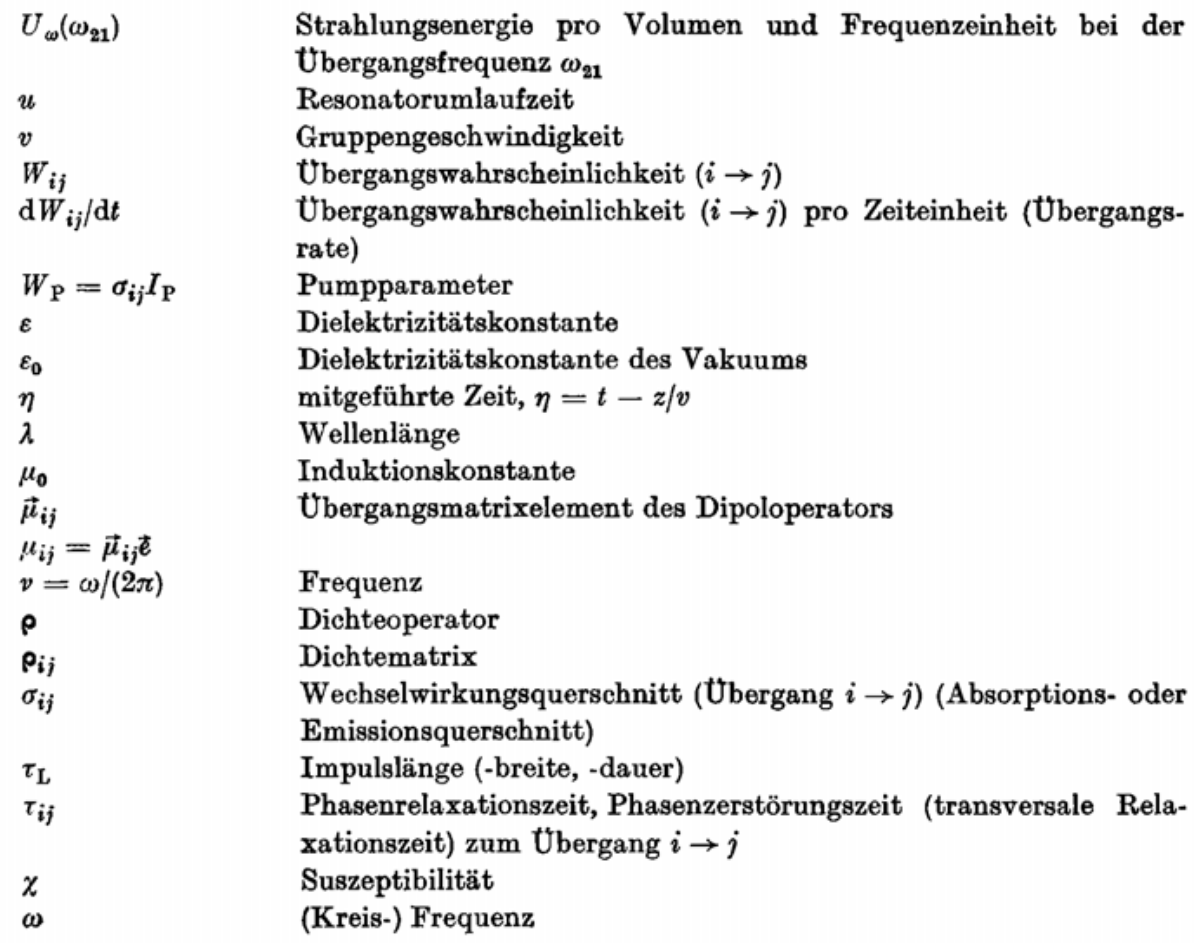

